\title{
The effects of $\mathrm{Mn}(\mathrm{III})$ ortho $\mathrm{N}$-alkylpyridyl- and diortho $\mathrm{N}, \mathrm{N}^{\prime}$ - imidazolylporphyrins on Oxygen and Glucose Deprivation - Induced Cell Death in Mixed Neuronal/Glial Cortical Cell Cultures
}

\author{
LISA WISE-FABEROWSKI ${ }^{1}$, DAVID S. WARNER ${ }^{2}$, IVAN SPASJOVIC ${ }^{3}$, and INES BATINIC- \\ HABERLE ${ }^{4}$ \\ ${ }^{1}$ Assistant Professor, Department of Anesthesiology, Duke University Medical Center \\ 2 Professor, Departments of Anesthesiology, Neurobiology, and Surgery, Duke University Medical \\ Center \\ ${ }^{3}$ Department of Medicine, Duke University Medical Center \\ ${ }^{4}$ Assistant Professor, Department of Radiation Oncology, Duke University Medical Center
}

\section{Abstract}

In vivo investigations have confirmed the beneficial effects of hydrophilic, cationic Mn(III) -based catalytic antioxidants in models of oxidative stress. We hypothesized the octyl porphyrin, MnTnOct-2-PyP ${ }^{5+}$, a lipophilic but equally potent antioxidant, would be more efficacious in reducing oxygen and glucose deprivation (OGD)-induced cell death. Using a cell culture model of rat mixed neuronal/glial cells, we investigated the effect of MnTnOct-2-PyP ${ }^{5+}$ on the OGDinduced cell death as compared to the effects of widely studied hydrophilic analogues MnTE-2$\mathrm{PyP}^{5+}$ and MnTDE-2-ImP ${ }^{5+} \mathrm{P}$ and a standard compound, dizocilpine (MK-801). Cell death was evaluated at 24 hours using lactate dehydrogenase (LDH) release, 3-(4,5-dimethyltiazol-2-yl) -2,5 diphenyltetrazolium bromide(MTT) and propidium iodide staining. At lower concentrations, all three porphyrins reduced cell death as compared to cultures exposed to OGD alone. When the cultures were exposed to MnTnOct-2-PyP ${ }^{5+}$ before OGD but not during the deprivation, it was a very efficacious compound as judged by LDH release. MnTnOct-2-PyP ${ }^{5+}$ becomes less efficacious if the exposure was prolonged. While no extensive toxicity was seen with MnTnOct-2$\mathrm{PyP}^{5+} \mathrm{P}$, the effects observed, though, might have been the result of the interplay of efficacy and toxicity leading to a diminished protectiveness.

\section{Keywords}

MnTE-2-PyP ${ }^{5+}$ (AEOL10113); MnTnOct-2-PyP ${ }^{5+}$; MnTDE-2-ImPP5+ (AEOL10150); neuronal/ glial cell culture; oxygen and glucose deprivation (OGD)

\section{Introduction}

Oxidative stress is a key intracellular pathological condition that mediates neuronal death in the presence of oxygen and glucose deprivation (OGD) [1]. Recent pharmacological advances have allowed for the design of different SOD mimics such as Mn salen derivatives [2], Mn cyclic polyamines [3], nitroxide [4], MitoQ series of compounds [5,6], and Mn

Address Correspondence to: Lisa Wise-Faberowski, MD, University of Colorado at Denver Health Sciences Center, Department of Anesthesiology, 13123 East $16^{\text {th }}$ Avenue, Aurora, CO 80045, Box 090Tel: 720-777-6224; Fax: (720) 777-7122

lisa.faberowski@uchsc.edu. 
porphyrins [7-10]. Most of them scavenge/reduce peroxynitrite, though, also with different efficacy, Mn porphyrins being the most efficacious $\log \mathrm{k}_{\text {red }} \geq 7.5$ [7-10]. We have shown that $\mathrm{Mn}$ (III) ortho isomeric positively charged $N$-alkylpyridyl- or $N, N^{\prime}$ -

dialkylimidazolylporphyrins (MnTalkyl-2-PyP ${ }^{5+}$, MnTDalkyl-2-ImP ${ }^{5+}$ ) are among the most effective synthetic antioxidants in scavenging both superoxide and peroxynitrite [11,12]. They offer remarkable protection in all diseases that have oxidative stress in common including central nervous system injuries such as ALS, cancer, diabetes, morphine tolerance [13-18]. MnTE-2-PyP ${ }^{5+}$ and MnTDE-2-ImP ${ }^{5+}$ were beneficial in middle cerebral artery occlusion models when given as late as $6 \mathrm{~h}$ after the insult [19].

We have recently reported that if catalytic potency in scavenging superoxide and peroxynitrite is maintained, but lipophilicity is increased, the efficacy in vivo increases tremendously [20]. We increased lipophilicity by increasing the length of the $N$-alkylpyridyl chains from 1 to 8 carbon atoms which increased liopophilicity up to 9-fold [Table 1] [20]. Thus, MnTnHex-2-PyP ${ }^{5+}$ that is 4.3-fold more lipophilic than MnTE-2-PyP ${ }^{5+}$, is 120 -fold more protective; it is efficacious in several models of oxidative stress (ALS, E. coli, kidney ischemia/reperfusion, morphine tolerance, radioprotection) at lowest level $(0.05 \mathrm{mg} / \mathrm{kg})$ among synthetic antioxidants tested[14,21-24]

Herein for the first time we tested the octyl analogue, MnTnOct-2-PyP ${ }^{5+}$ (Figure 1, Table 1\} in a mammalian system of oxidative stress. MnTnOct-2-PyP ${ }^{5+}$ was as effective as hexyl in protecting SOD-deficient $E$. coli to grow aerobically [21]. Both hexyl and octyl porphyrins have partial micellar character and are thus toxic at higher doses. Yet the $\mathrm{TD}_{50}$ determined for mice to be $12.5 \mathrm{mg} / \mathrm{kg}$ (subcutaneously) is 250 higher than its effective dose of $0.05 \mathrm{mg} /$ $\mathrm{kg}$ while that ratio is 15 with MnTE-2-PyP ${ }^{5+}$ allowing thus wider therapeutic window with MnTnHex-2-PyP ${ }^{5+}$ [Moen Panni unpublished]. The same is likely valid with MnTnOct-2$\mathrm{PyP}^{5+}$; therefore this compound may be regarded a prospective therapeutic also.

The enhanced bioavailability of MnTnOct-2-PyP ${ }^{5+}$ should promote improved neuronal survival as compared to hydrophilic compounds with fewer carbon atoms in the alkylpyridyl chains $[20,25,26]$. This hypothesis was tested in mixed neuronal/glial cortical cell cultures exposed to oxygen and glucose deprivation.

\section{Methods}

All animal procedures were approved by the Duke University Animal Care and Use Committee.

\section{Preparation of mixed neuronalglial cell cultures}

Mixed neuronal/glial cultures were prepared from fetal Sprague-Dawley (Harlan Sprague Dawley, Inc. Indianapolis, IN) rat brains at 18 days of gestation as previously described [wrong ref]. Brains were harvested from 10-15 pups and dissected to separate cortex from meninges and subcortical structures using anatomical landmarks. Cortices were pooled and minced into $2 \mathrm{~mm}^{3}$ pieces in a buffered salt solution (BSS; Hanks Balanced Salt Solution [Life Technologies, Gaithersburg, MD] supplemented with $20 \mathrm{mM}$ HEPES buffer (pH 7.4, containing $0.25 \%$ trypsin [Life Technologies]). The tissue was incubated for $20 \mathrm{~min}$ at $37^{\circ} \mathrm{C}$ in a $5 \% \mathrm{CO}_{2} / 95 \%$ room air atmosphere, then washed twice with ice-cold, glutamine-free minimum essential medium (MEM; Life Technologies) containing $15 \mathrm{mM}$ glucose, $5 \%$ fetal bovine serum (Gibco Diagnostics, Inc., Madison, WI), 5\% horse serum (GIBCO), and 1\% DNase-I (Sigma Chemical Co., St. Louis, MO, U.S.A.). Tissue pieces were dissociated by trituration through a fire-polished 9" Pasteur pipette. The resultant suspension was centrifuged at $50 \mathrm{~g}$ for $10 \mathrm{~min}$, the supernatant discarded, and the pellet resuspended in growth medium (MEM supplemented with $15 \mathrm{mM}$ glucose, 5\% fetal bovine serum, and 5\% 
horse serum). The dissociated cells were plated to achieve a confluent monolayer $\left(4 \times 10^{5}\right.$ cells per well for neuronal/glial cultures) on poly-D-lysine coated, 24-well culture plates (Falcon 3047; Becton Dickinson Co., Lincoln Park, NJ). Cultures were maintained undisturbed at $37{ }^{\circ} \mathrm{C}$ in a humidified $5 \% \mathrm{CO}_{2}$ /balance room air atmosphere for 10-14 days prior to use. Previous studies performed under identical culture conditions demonstrated cell types in 10 day old cultures are $54 \pm 4 \%$ neurons and $46 \pm 7 \%$ glia as determined by immunohistochemical staining for cell specific cytoskeletal filaments (neurofilament-160 for neurons and glial fibrillary acidic protein for astrocytes)[27].

\section{Preparation of Mn porphyrins}

All compounds studied are synthesized as previously reported in details [7,20,28]. Particular attention was paid to their purification, especially to a longer alkyl-chain analogue, MnTnOct-2-PyP ${ }^{5+} \mathrm{P}$, which was additionally purified using ultrafilter 500-cutoff.

\section{Dose-response}

Based on SOD-deficient E. coli study, a dose response curve was determined using 3, 10, 30 and $100 \mu \mathrm{M}$ of MnTE-2-PyP ${ }^{5+}$ and MnTDE-2-ImPP ${ }^{5+}$ [21]. Identical concentrations were chosen for the MnTnOct-2-PyP ${ }^{5+}$. All compounds were diluted in glucose-free phosphate buffered saline (PBS: $7.65 \mathrm{~g} \mathrm{NaCl}, 0.724 \mathrm{~g} \mathrm{Na} 2 \mathrm{OH} 4$ and $0.21 \mathrm{~g} \mathrm{~K} 2 \mathrm{PO} 4, \mathrm{pH} 7.4$ ) to provide the desired concentrations and immediately used for experimentation.

\section{Oxygen and glucose deprivation}

The original glucose containing media was removed from all treatment groups and replaced with a glucose-free phosphate buffered saline (PBS: $7.65 \mathrm{~g} \mathrm{NaCl}, 0.724 \mathrm{~g} \mathrm{Na}_{2} \mathrm{OH}_{4}$ and 0.21 $\mathrm{g} \mathrm{K}_{2} \mathrm{PO}_{4}, \mathrm{pH}$ 7.4). All media changes were followed by a wash with PBS. No serum was included in the glucose-free PBS. In the oxygen and glucose deprivation phase, the media was washed with PBS and changed to hypoxic, glucose-free PBS. The glucose free/hypoxic PBS was prepared by passing the PBS through a microbubbler apparatus containing the hypoxic $\left(94 \% \mathrm{~N}_{2} / 6 \% \mathrm{CO}_{2}, \mathrm{pH} 7.37+/-0.4\right)$ gas mixture. The $94 \% \mathrm{~N}_{2} / 6 \% \mathrm{CO}_{2}$ gas mixture was used to maintain the previous incubating conditions without oxygen and with minimal change in $\mathrm{pH}$. The hypoxic, glucose-free PBS was then applied in a thin layer (enough to cover the cells) to the cell culture dishes. Dishes exposed to hypoxia were then placed in a small, 3-liter, airtight experimental hypoxia chamber (Billups-Rothenberg; San Diego, CA) with inflow and outflow connectors. The experiments were conducted in a constant $37^{\circ} \mathrm{C}$ environment by placing the chambers in a water-jacketed incubator. The gaseous environment was controlled by the delivery of all gas via a heater humidifier (FisherPaykel; Laguna Hills, CA) servo-controlled to $37^{\circ} \mathrm{C}$ via the inflow adapter of the chamber. Delivered and end-tidal concentrations of oxygen were monitored using a gas analyzer (Datex Instruments Corporation, Tewksbury, MA) and maintained at $<0.2 \%$ [29].

As performed previously [13], cultures were exposed to conditions of oxygen and glucose deprivation for $2 \mathrm{~h}$. The exposure was terminated by removing the PBS, adding the original media for $24 \mathrm{hrs}$, and then analyzing for cell death and viability as described below. Control cells were incubated in PBS in a normoxic incubator for the same time period as the experimental group.

\section{LDH release}

Cellular injury was assessed 24 hours after oxygen and glucose deprivation by measuring the amount of LDH released into overlying medium by damaged cells. In brief, a $200-\mu \mathrm{l}$ sample of culture medium was added to a polystyrene cuvette containing $10 \mathrm{mM}$ lactate and $5 \mu \mathrm{mol}$ of NAD ( $B$-nicotinamide adenine dinucleotide; Sigma, St. Louis, Mo.) in $2.75 \mathrm{ml}$ of 
$50 \mathrm{mM}$ glycine buffer $\mathrm{pH} 9.2$ at $24^{\circ} \mathrm{C}$. LDH activity was determined from the initial rate of reduction of $\mathrm{NAD}^{+}$as calculated using a linear least square curve fit of the temporal changes in fluorescence signal from the cuvette ( $340 \mathrm{~nm}$ excitation, $450 \mathrm{~nm}$ emission) and expressed in units of enzymatic activity ( $\mathrm{nmol}$ of lactate converted to pyruvate per min). Analysis was performed on a fluorescence spectrophotometer (Perkin Elmer Model LS50B;

Bodenseewerk GmbH, Uberlinger, Germany).

\section{Cell viability assay}

Twenty-four hours after treatment, the neuronal/glial cell cultures were incubated with 250 $\mu \mathrm{g} / \mathrm{ml}$ 3-(4, 5-dimethyltiazol-2-yl) -2, 5 diphenyltetrazolium bromide (MTT, Sigma)) for 30 $\mathrm{min}$ at $37^{\circ} \mathrm{C}$ [30]. MTT is absorbed into cells and transformed into formazan by mitochondrial succinate dehydrogenase. Accumulation of formazan directly reflects the activity of mitochondria, which functions as an indirect measurement of cell viability. Sodium dodecyl sulfate in dilute hydrochloric acid was used to dissolve the purple formazan product. After the precipitate in each well was resuspended on a microplate mixer for 10 min, an optical density (OD) reading at $540 \mathrm{~nm}$ was measured using an ELISA plate reader. A dose response curve was determined and cell viability measured as absorbance was compared to control and cell cultures exposed to oxygen and glucose deprivation.

\section{Propidium iodide assay}

Cells were exposed to a final concentration of $40 \mu \mathrm{g} / \mathrm{ml}$ of propidium iodide dissolved in BSS for 10 min. After washing with BSS, the cells were fixed with $4 \%$ paraformaldehyde for $10 \mathrm{~min}$, followed by another washing with BSS. The cells were then stored in $300 \mu \mathrm{l}$ of BSS. All staining procedures were done at room temperature. The cultures were then viewed under an inverted fluorescent microscope at 20x magnification with the observer blinded to treatment condition. Three fields were chosen in each separate well (10 wells for each experimental condition) for cell count determination per experiment. Dead cells were defined as those visibly stained by propidium iodide, which signals membrane disruption [27].

\section{Statistical Analysis}

Data were compared by one-way analysis of variance. When indicated by a significant $F$ ratio, post hoc testing was performed by use of Scheffe's test. Values are reported as mean \pm $\mathrm{SD}$. A $\mathrm{P}$ value $<0.05$ was considered significant. Statistical analysis was performed using StatView 5.0 (SAS; Cary, NC).

\section{Results}

The protection afforded against oxygen and glucose deprivation-induced cell death was afforded with all compounds (Figures 2-4,6). Differences observed relate to the levels of those compounds and the type of assay used. All the compounds are more beneficial if the pretreatment of the cells before oGD was performed (Figure 2 vs Figure 3). The most effective compound as judged by LDH assay (when treatment with compound was done before and during deprivation) was MnTE-2-PyPP ${ }^{5+}$ (Figure 3), while MnTDE-2-ImP ${ }^{5+}$ was the most protective judged by propidium iodide assay (Figure 5). MnTnOct-2-PyP ${ }^{5+} \mathrm{P}$ was efficacious in LDH assay when cell were treated prior to stress only; during prolonged exposure potential toxicity counterbalanced the protectiveness (Figure 2).

\section{LDH analysis}

Two experiments were used to determine neuronal/glial cell death using LDH analysis. These experiments are the following: 1) 30 min pretreatment with the Mn porphyrins prior 
to oxygen and glucose exposure (Figure 2). 2); and 30 min pretreatment with continuation of the treatment during the $2 \mathrm{~h}$ of oxygen and glucose deprivation (Figure 3 ). In both experiments, the conditions provided by $2 \mathrm{~h}$ of oxygen and glucose deprivation were statistically different than control and $10 \mu \mathrm{M}$ MK801 to give the study a power of 1.0. Six wells were used for all conditions in both experiments.

In the first experiment, all compounds at all concentrations statistically decreased oxygen and glucose deprivation-induced LDH release ( $<<0.001)$, except for $3 \mu \mathrm{M} \mathrm{MnTDE}^{2}-\mathrm{ImP}^{5+}$, 10 and $30 \mu \mathrm{M}$ of the MnTnOct-2-PyP $\mathrm{P}^{5+}$. When compared to control conditions, LDH release was similar in the $100 \mu \mathrm{M}$ MnTE-2-PyP ${ }^{5+}$, and MnTnOct-2-PyP ${ }^{5+}$, and 3 and $30 \mu \mathrm{M}$ MnTE-2-PyP ${ }^{5+}$ groups ( $\left.\mathrm{p}>0.999\right)$.

In the second experiment, when compared to control conditions, $30 \mu \mathrm{M}$ MnTE-2-PyP ${ }^{5+}$ $(\mathrm{p}=0.296)$ demonstrated similar LDH release. When compared to cells stressed by oxygen and glucose deprivation, statistical significance was achieved with 3,10 and $30 \mu \mathrm{M}$ MnTnOct-2-PyP ${ }^{5+}$, MnTE-2-PyP ${ }^{5+}$ and MnTDE-2- $\mathrm{ImP}^{5+} \mathrm{P}$,

\section{Cell Viability Assay}

Two hours of oxygen and glucose deprivation provided a $(\mathrm{p}<0.001)$ statistical difference in cell viability to fulfill a power of 1.0 for this assay (Figure 4). Ten wells for each condition were used for this assay. None of the conditions, including $10 \mu \mathrm{M}$ MK801, afforded statistically significant improved cell viability when compared to the cells that underwent oxygen and glucose deprivation alone.

\section{Propidium lodide Staining}

Ten cell culture wells were used for each of the experimental conditions to provide a power of 1.0 (Figure 5). When compared to control, the number of dead cells was statistically different $(\mathrm{p}<0.001)$. Compared to oxygen and glucose deprivation alone, $10 \mu \mathrm{M} \mathrm{MK} 801$ and $10 \mu \mathrm{M}$ of MnTDE-2-ImP ${ }^{5+} \mathrm{P}$, afforded significant protection against cell death $(\mathrm{p}<0.001)$.

Cell death was enhanced with 30 and $100 \mu \mathrm{M}$ concentrations of MnTDE-2-ImP ${ }^{5+}$. The other conditions had no statistically significant effect. There was a trend towards protection with low, $3 \mu \mathrm{M}$ MnTnOct-2-PyPP ${ }^{5+}$, indicating that this compound is likely very effective at much lower doses where toxicity will not counterbalance benefit.

\section{Discussion}

Superoxide dismutases are first lines of defense maintaining steady state levels of superoxide, thus all other reactive species formed down stream, particularly peroxynitrite also. MnSOD (SOD2) is one of the four major superoxide dismutases and is distributed in the mitochondrial matrix [wrong refs, must come some review refs maybe from Fridovich] and abundant in neural tissue. Under pathological conditions endogenous SOD may not be able to offer sufficient protection. Thus, exogenous antioxidants may be beneficial. Several different classes have been studied, metalloporhyrins being among the most effective ones [2-10,31].

We have developed structure-activity relationship for Mn porphyrins. Based on structural activity relationship it became obvious that the most potent compounds must have Mn site sufficiently electron-deficient for thermodynamic facilitation of the $\mathrm{O}_{2}{ }^{--}$dismutation. The efficacy to remove superoxide parallels the efficacy to reduce peroxynitrite (12). Further, the respective compounds must bear positive charges close to the $\mathrm{Mn}$ site needed for the electrostatic facilitation for the approach of negatively charged $\mathrm{O}_{2}{ }^{--}$and $\mathrm{ONOO}^{-}[8,10]$. The proper lipophilicity and the size of molecule are also critical for in vivo bioavailabity, i.e. efficacy $[14,20-24,32,33]$. Finally the toxicity needs to be modulated as well [32]. 
Our most potent compounds showed efficacy in nearly any model of oxidative stress tested, including central nervous system injuries [13-18,20-24]. MnTE-2-PyP ${ }^{5+}$ and MnTDE-2$\mathrm{ImP}^{5+} \mathrm{P}$ have demonstrated neuroprotection in stroke model and are associated with a decrease in aconitase inactivation, 8-hydroxyguanine formation and cytokine expression $[13,19]$. Based on the remarkable in vivo efficacy, the lipophilic MnTnHex-2-PyP ${ }^{5+}$ is presently our most promising drug. Although it suffers from micellar-based toxicity at higher dose its toxicity to efficacy ratio is 250 and 17 -fold better than for its more hydrophilic analogue, MnTE-2-PyP ${ }^{5+}$. Herein, for the first time we tested the octyl analogue, $\mathrm{MnTnOct}-2-\mathrm{PyP}^{5+}$ on efficacy and toxicity. It is 1.4-fold more lipophilic than MnTnHex-2-PyP ${ }^{5+}$ compound.

The efficacy observed is comparable to or less than that of other two Mn porphyrins with fewer carbon atoms in the alkyl chains. The effects observed were dependent upon the assay and the concentrations used. MTT assay was not able to distinguish between compounds. In the LDH assay, where treatment only prior to oxygen and glucose deprivation was done, 100 $\mu \mathrm{M}$ MnTnOct-2-PyP ${ }^{5+}$ was efficacious, decreasing the injury of cells to control levels. The protective effects of $\mathrm{MnTnOct}-2-\mathrm{PyP}^{5+}$ were lesser than expected as judged by propidium iodide and with LDH assay (where pretreatment and treatment during deprivation was performed), likely hampered by the toxicity involved when cell exposure to the drug was prolonged; i.e. the effects of MnTnOct-2-PyP ${ }^{5+}$ observed might have resulted from the interplay of efficacy and toxicity.

Still, given the very sensitive nature of neurons, the toxic effects, if any, are significantly lower than we expected. That is a motivating observation with respect to the future studies on the evaluation of the utility of MnTnOct-2-PyP ${ }^{5+}$ as a neuroprotective agent. In all 4 assays, MnTE-2-PyP ${ }^{5+}$ and MnTDE-2-ImP ${ }^{5+} \mathrm{P}$ were of comparable efficacy. Judged by LDH assay the former was a better performer while based upon the propidium iodide assay the latter was performing better. All compounds show signs of toxicity at higher doses. Our findings are consistent with those obtained previously by Sheng et al. on the effect of MnTDE-2-ImPP ${ }^{5+}$ in mixed neuronal/glial cell cultures exposed to $2 \mathrm{hr}$ of oxygen and glucose deprivation.

The failure of compounds to show protective effects by MTT assay when compared to propidium iodide may be in part related to the different nature of these two assays one being extracellular (LDH) and the other intracellular (MTT) [24]. With MTT assay a trend towards protection of cells was observed with MnTE-2-PyP ${ }^{5+}$. Oxygen and glucose deprivation induced mitochondrial aconitase activation $[1,13,14]$, may have provided greater insight into the protection afforded by MnTnOct-2-PyP ${ }^{5+}$ at the mitochondrial level. Our data $(33,34)$ indicate that MnTE-2-PyP ${ }^{5+}$ accumulates in all organs, brain included and in mitochondria and nucleus (Spasojevic, Tse, Piganelli unpublished). It is highly likely that octyl analogue distributes at greater extent in all organs and subcellular compartments. Our most recent data that have shown the remarkable benefit of lipophilic Mn porphyrins in vivo in animal models, justify studies that are in progress addressing their organ and cellular distribution.

\section{Acknowledgments}

LWF thanks the Multidisciplinary Neuroprotection Laboratories at Duke University Medical Center (NIH Grants T32 GM08600-09 and RO1 GM067139-03); IBH acknowledges the support by the National Institutes of Health (IR21-ESO/3682) and the National Institutes for Allergy and Infectious Diseases (U19AI067798) grants; IS thanks NIH/NCI Duke Comprehensive Cancer Center Core Grant (5-P30-CA14236-29); the authors wish to thank Ms. Candace Berryman for her editorial assistance. 


\section{Abbreviations}

\author{
MnTE-2-PyP ${ }^{5+}$ \\ MnTnOct-2-PyP ${ }^{5+}$ \\ LDH \\ MTT \\ SOD
}

$\mathrm{Mn}(\mathrm{III})$ mesotetrakis(N-ethylpyridinium-2-yl)porphyrin, EPyP and MnTDE-2-ImPP ${ }^{5+} \mathrm{Mn}(\mathrm{III})$ mesotetrakis(N,N'diethylpyridinium-2-yl)porphyrin, DEImP

\author{
$\mathrm{Mn}(\mathrm{III})$ mesotetrakis(N-n-octylpyridinium-2-yl)porphyrin, OctPyP \\ lactate dehydrogenase \\ 3-(4,5-dimethyltiazol-2-yl) -2,5 diphenyltetrazolium bromide \\ superoxide dismutase
}

\section{References}

1. Li QY, Pedersen C, Day BD, Patel M. Dependence of excitotoxic neurodegeneration on mitochondrial aconitase inactivation. J of Neurochem. 2001; 78:746-755. [PubMed: 11520895]

2. Van Empel VPM, Bertrand AT, Van Oort RJ, Van der Nagel R, Engelen M, Van Rijen HV, Doevendans PA, Crijns HJ, Ackerman SL, Sluiter W, De Windt LJ. EUK_8, a superoxide dismutase and catalase mimetic, reduces cardiac oxidative stress and ameliorates pressure overloadinduced heart failure in the harlequin mouse mutant. J Am Coll Cardiol. 2006; 48:8245-832.

3. Salvemini D, Wang Z-Q, Zweier JL, Samouilov A, Macarthur H, Misko TP, Curie MG, Cuzzocrea S, Sikorski JA, Ruley DP. A nonpeptidyl mimic of superoxide dismutase with therapeutic activity ion rats. Science. 1999; 286:304-306. [PubMed: 10514375]

4. Goldstein S, Samuni A, Hideg K, Merenyi G. Structure-activity relationship of cyclic nitroxides as SOD mimics and scavengers of nitrogen dioxide and carbonate radicals. J Phys Chem A. 2006; 110:3679-3685. [PubMed: 16526651]

5. James AM, Cocheme HM, AJ, Murphy MP. Interactions ofSmith, R. mitochondria-targeted and untargeted ubiquinones with the mitochondrial respiratory chain and reactive oxygen species. J Biol Chem. 2005; 28:21295-21312. [PubMed: 15788391]

6. Smith RAJ, Porteous CM, Ganes AM, Murphy MP. Delivery of bioactive molecules to mitochondria. Proc Natl Acad Sci. 2003; 100:5407-5412. [PubMed: 12697897]

7. Batini -Haberle I, Benov L, Spasojevi I, Hambright P, Crumbliss AL, Fridovich I. The relationship between redox potentials, proton dissociation constants of pyrrolic nitrogens, and in vitro and in vivo superoxide dismutase activities of Manganese(III) and Iron(III) cationic and anionic porphyrins. Inorg Chem. 1999; 38:4011-4022.

8. Rebouças JS, Spasojevi I, Batini -Haberle I. Pure manganese(III) 5,10,15,20-tetrakis(4-benzoic acid)porphyrin (MnTBAP) is not a superoxide dismutase mimic in aqueous systems: A case of structure-activity relationship as a watchdog mechanism in experimental therapeutics and biology. $\mathrm{J}$ Inorg Biol Chem. 2008; 13:289-302.

9. Spasojevi I, Batini -Haberle I, Stevens RD, Hambright P, Thorpe AN, Grodkowski J, Neta P, Fridovich I. Manganese(III) biliverdin IX dimethyl ester: A powerful catalytic scavenger of superoxide employing the $\mathrm{Mn}(\mathrm{III}) / \mathrm{Mn}(\mathrm{IV})$ redox couple. Inorg Chem. 2001; 40:726-739. [PubMed: 11225116]

10. Spasojevic I, Batinic-Haberle I, Reboucas JS, Idemori YM, Fridovich I. Electrostatic Contribution in the Catalysis of $\mathrm{O}_{2}{ }^{--}$Dismutation by Superoxide Dismutase Mimics. J Biol Chem. 2003; 278:6831-6837. [PubMed: 12475974]

11. Ferrer-Sueta G, Batinic-Haberle I, Spasojevic I, Fridovich I. Peroxynitrite Scavanging by Manganese (III) Meso-Tetrakis-( $N$-methylpyridyl)Porphyrins. Chem Res Toxicol. 1999; 12:442449. [PubMed: 10328755]

12. Ferrer-Sueta G, Vitturi D, Batinic-Haberle I, Fridovich I, Goldstein S, Czaspki G, Radi R. Reactions of manganeses porphyrins with peroxynitrite and carbonate radical ion. J Biol Chem. 2008; 278:27432-27438. [PubMed: 12700236] 
13. Sheng H, Enghild JJ, Patel M, Batinic-Haberle I, Calvi C, Day BJ, Pearlstein RD, Crapo JD, Warner DS. Effects of metalloporphyrin catalytic antioxidants in experimental brain ischemia. Free Radical Biology and Medicine. 2002; 33:942-961.

14. Gauter-Fleckenstein B, Fleckenstein K, Owzar K, Jian C, Batinic-Haberle I, Vujaskovic Z. Comparison of two Mn porphyrin-based mimics of superoxide-dismutase (SOD) in pulmonary radioprotection. Free Radic Biol Med. 2007 published on-line Nov.

15. Sheng H, Batinic-Haberle I, Warner DS. Catalytic Antioxidants as Novel Pharmacologic Approaches to Treatment of Ischemic Brain Injury. Drug News and Perspectives. 2002; 15:654665. [PubMed: 12677251]

16. Piganelli JD, Flores SC, Cruz C, Koepp J, Young R, Bradley B, Kachadourian R, Batinic-Haberle I, Haskins K. A Metalloporphyrin Superoxide Dismutase Mimetic (SOD Mimetic) Inhibits Autoimune Diabetes. Diabetes. 2002; 51:347-355. [PubMed: 11812741]

17. Moeller BJ, Batinic-Haberle I, Spasojevic I, Rabbani ZN, Anscher MS, Vujaskovic Z, Dewhirst MW. Effects of a catalytic metalloporphyrin antioxidant on tumor radioresponsiveness. Int J Rad Oncol Biol Phys. 2005; 63:545-552.

18. Sompol P, Ittarat W, Tangpong J, Chen Y, Doubinskaia I, Batinic-Haberle I, Mohammad Abdul H, Butterfield A, St Clair DK. Alzheimer's disease: An insight into the mechanisms of oxidative stress-mediated mitochondrial injury. Neuroscience. 2008 in press.

19. Mackensen GB, Patel M, Sheng H, Calvi C, Batinic-Haberle I, Day BJ, Liang LP, Fridovich I, et al. Neuroprotection from delayed post-ischemic administration of a metalloporphyrin catalytic antioxidant. J Neurosci. 2001; 21:4582-4592. [PubMed: 11425886]

20. Batinic-Haberle I, Spasojevic I, Stevens RD, Hambright P, Fridovich IJ. Manganese(III) Meso Tetrakis Ortho $\mathrm{N}$-alkylpyridylporphyrins. Synthesis, Characterization and Catalysis of $\mathrm{O}_{2}{ }^{--}$ Dismutation. Chem Soc, Dalton Trans. 2002:2689-2696.

21. Okado-Matsumoto A, Batinic-Haberle I, Fridovich I. Complementation of SOD-deficient Escherichia Coli by manganese porphyrin mimics of superoxide dismutase activity. Free Radic Biol Med. 2004; 37:401-10. [PubMed: 15223074]

22. Saba H, Batini -Haberle I, Munusamy S, Mitchell T, Lichti C, Megyesi J, MacMillan-Crow LA. Manganese porphyrin reduces renal injury and mitochondrial damage during ischemia/reperfusion. Free Radic Biol Med. 2007; 42:1571-1578. [PubMed: 17448904]

23. Crow J. MnTnHex-2-PyP ${ }^{5+}$ in ALS. unpublished.

24. Batibic-Haberle, I.; Ndengele, MM.; Cuzzocrea, S.; Reboucas, JS.; Matuscak, GM.; St Clair, DK.; Dewhirst, MW.; Spasojevic, I.; Salvemini, D. Inhibition of morphine tolerance and suppression of tumor angiogenesis: Dual action of Mn porphyrin-based drugs in cancer therapy and pain management. AACR; San Diego: 2008.

25. Leinenweber SB, Sheng H, Lynch JR, Batinic-Haberle I, Laskowitz DT, Crapo JD, Pearlstein RD, Warner DS. Effects of a Manganese(III) Porphyrin Catalytic Antioxidant in a Murine Model of Closed Head Injury. Eur J Pharmacol. 2006; 531:126-132. [PubMed: 16455070]

26. Dugan LL, Gabrielsen JK, Yu SP, Lin TS, Choi DW. Buckminsterfullerenol free radical scavengers reduce excitotoxic and apoptotic cell death of cultured cortical neurons. Neurobiol Dis. 1996; 3:129-135. [PubMed: 9173920]

27. Wise-Faberowski, Aono M, Pearlstein Warner DS. Apoptosis is not enhancedR, in primary mixed neuronal/glial cultures protected by isoflurane against N-methyl-d-aspartate excitotoxicity. Anesth Analg. 2004; 99:1708-14. [PubMed: 15562059]

28. Batinic-Haberle I, Spasojevic I, Stevens RD, Hambright P, Neta P, Okado-Matsumoto A, Fridovich I. New Class of Potent Catalysts of $\mathrm{O}_{2}{ }^{--}$Dismutation. $\mathrm{Mn}(\mathrm{III})$ methoxyethylpyridyland methoxyethylimidazolylporphyrins. J Chem Soc Dalton Trans. 2004:1696-1702.

29. Wise-Faberowski, Sumners C, Raizada M. Oxygen and glucose deprivation-induced neuronal apoptosis is attenuated by halothane and isoflurane. Anesthesia and Analgesia. 2001; 93:1281-7. [PubMed: 11682415]

30. Koh SH, Noh MY, Kim SH. Amyloid-beta-induced neurotoxicity is reduced by inhibition of glycogen synthase kinase-3. Brain Res. 2008; 1188:254-62. [PubMed: 18031715]

31. Warner DS, Sheng H, Batinic-Haberle I. Oxidants, Antioxidants, and the Ischemic Brain. J Exp Biology. 2004; 207:3221-3231. 
32. Spasojevic I, Chen Y, Noel TJ, Fan P, Zhang L, Reboucas JS, St Clair DK, Batinic-Haberle I. Potent redox modulator of oxidative stress, MnTE-2-PyP ${ }^{5+}$. Pharmacokinetics in mouse plasma, liver, kidney, spleen, lung, heart and brain. Free Radic Biol Med. 2008 submitted.

33. Spasojevic I, Yumin C, Noel T, Yu I, Pole MP, Zhang L, Zhao Y, St Clair DK, Batinic-Haberle I. Mn porphyrin-based SOD mimic, MnTE-2-PyP ${ }^{5+}$ targets mouse heart mitochondria. Free Radic Biol Med. 2007; 42:1193-1200. [PubMed: 17382200] 

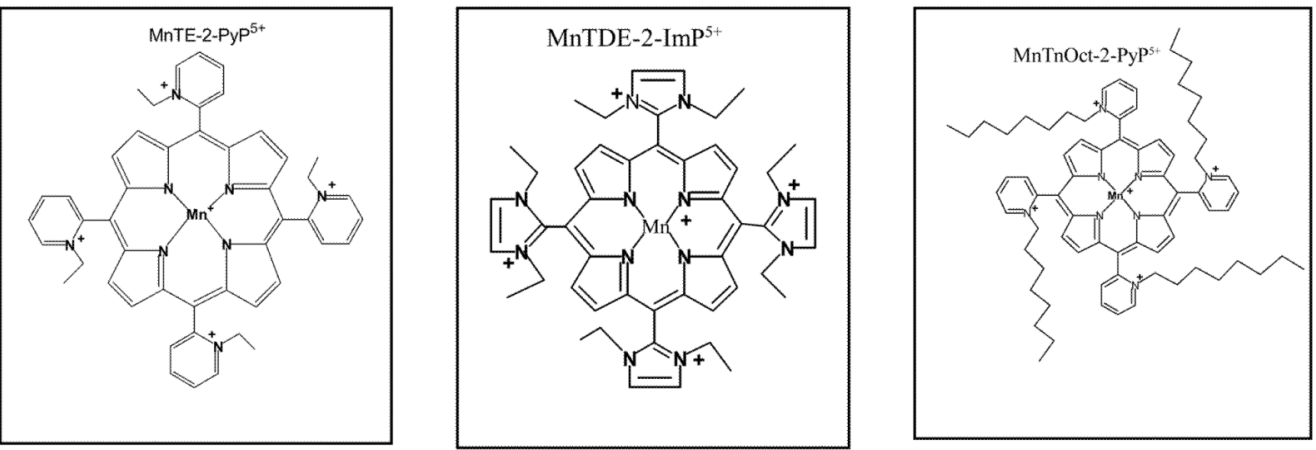

Figure 1.

Schematic drawings of cationic ortho $N \mathrm{Mn}$ (III) -alkylpyridyl- and diortho $N, N$ 'imidazolylporphyrin-based catalytic antioxidants MnTE-2-PyP ${ }^{5+}$ (AEOL10113) and

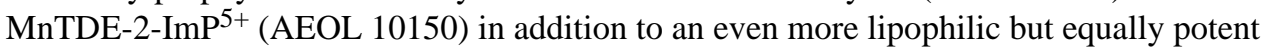
antioxidant, the octyl porphyrin, MnTnOct-2-PyP ${ }^{5+}$. 

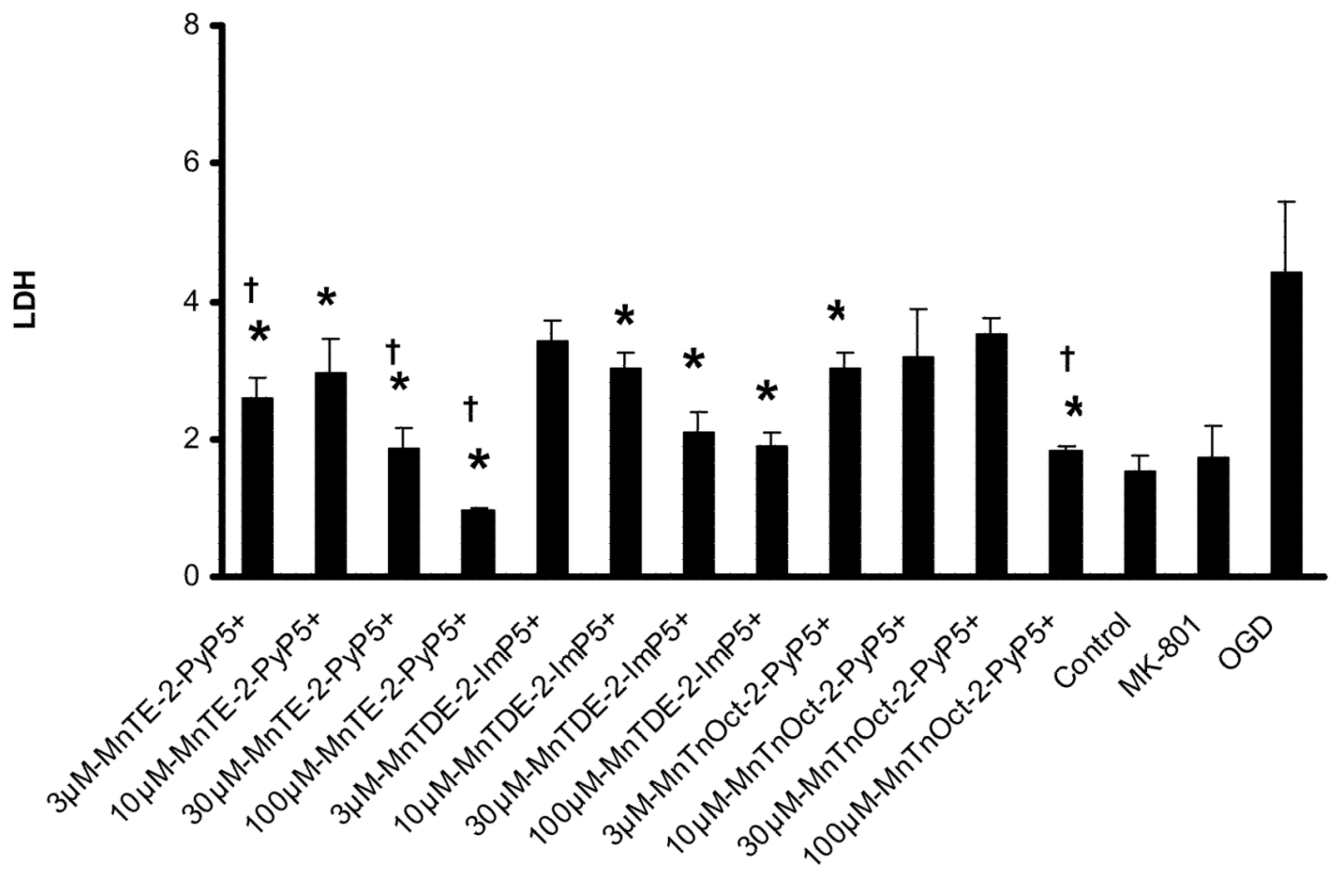

Figure 2. LDH dose response curve: 30 min pretreatment prior to exposure to oxygen and glucose deprivation

A dose response curve was determined at 24 hours after oxygen and glucose deprivation, using previously in vivo dosing (3, 10, 30 and $100 \mu \mathrm{M})$ of MnTE-2-PyP ${ }^{5+}$ and MnTDE-2$\mathrm{ImP}^{5+} \mathrm{P}$. The same doses were used for the MnTnOct-2-PyP $\mathrm{P}^{5+}$ compound. These conditions were compared to control, oxygen and glucose deprivation. * statisitical difference $(\mathrm{p}<0.005)$ from oxygen and glucose deprivation (OGD); $\dagger$ statisitically equal $(\mathrm{p}>0.999)$ to control. 


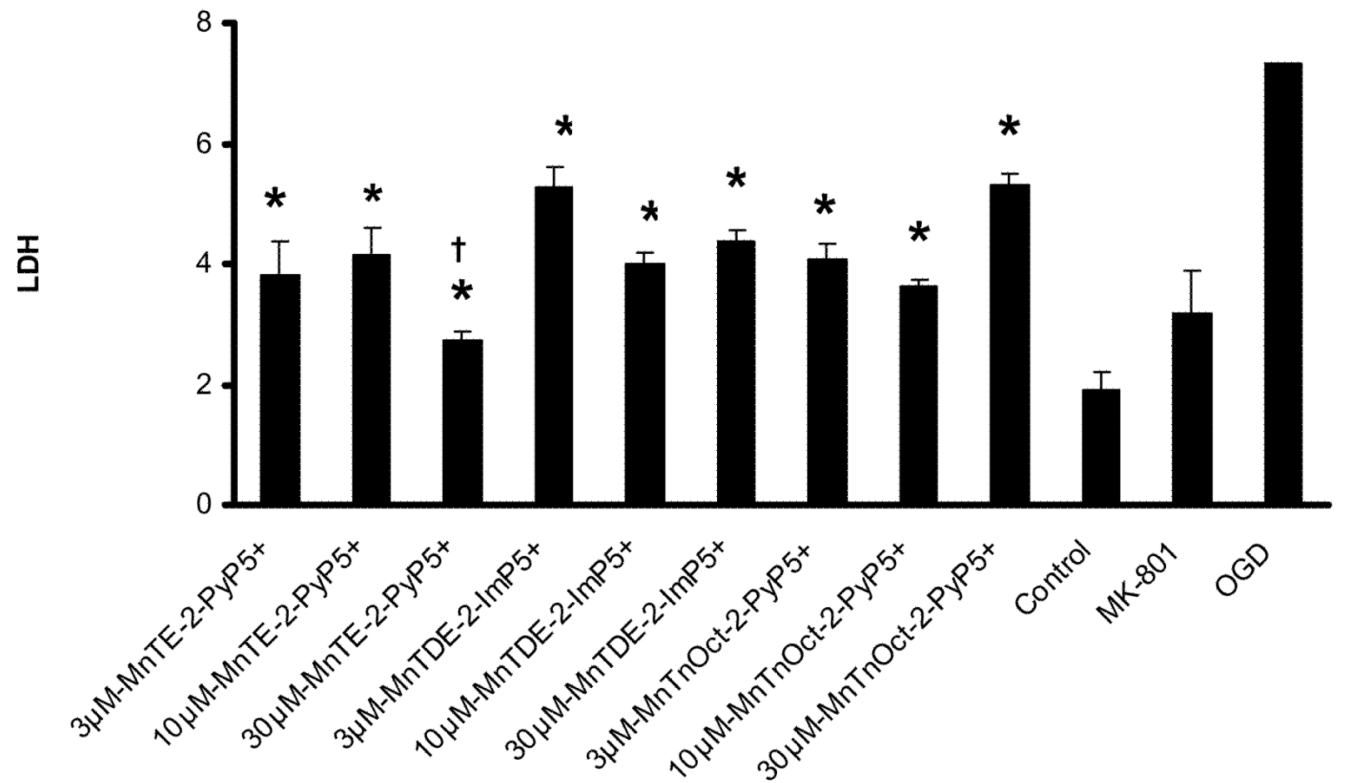

Figure 3. LDH dose response curve: 30 min pretreatment prior to exposure to oxygen and glucose deprivation with continued exposure during $\mathbf{2}$ hours of oxygen and glucose deprivation A dose response curve was determined at 24 hours after exposure to oxygen and glucose deprivation, using previously in vivo dosing $(3,10,30$ and $100 \mu \mathrm{M})$ of $\mathrm{MnTE}^{\mathrm{T}}-\mathrm{PyP}^{5+}$ and MnTDE-2-ImPP ${ }^{5+}$. . The same doses were used for the MnTnOct-2-PyP ${ }^{5+}$. These conditions were compared to control, oxygen and glucose deprivation alone and $10 \mu \mathrm{M}$ MK801. Six wells were used for each condition. * statisitical difference $(\mathrm{p}<0.005)$ from oxygen and glucose deprivation (OGD); $\uparrow$ statisitically equal $(\mathrm{p}>0.999)$ to control. 


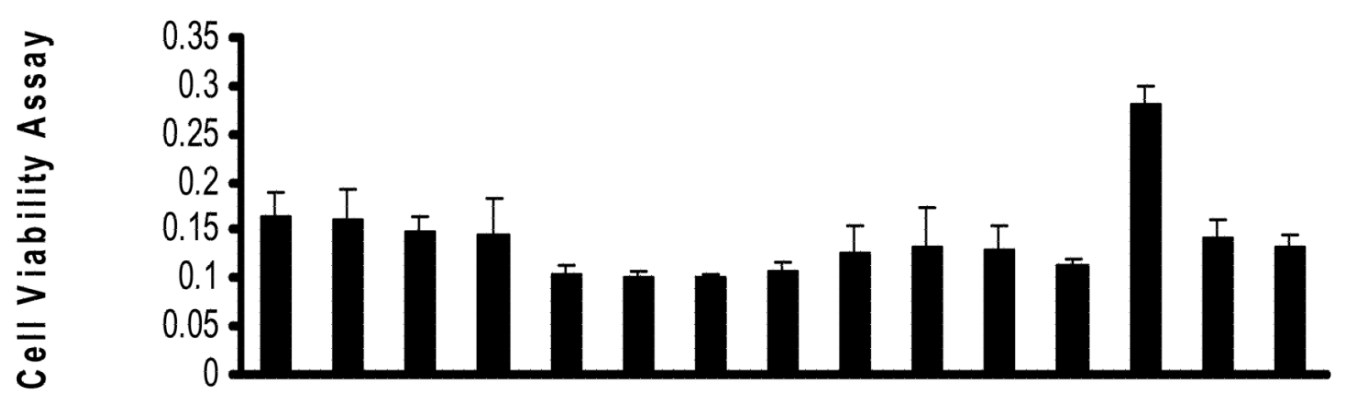

Figure 4. Cell viability/MTT assay: $30 \mathrm{~min}$ pretreatment prior to exposure to oxygen and glucose deprivation with continued exposure during 2 hours of oxygen and glucose deprivation Cell viability was measured 24 hours after exposure to the experimental conditions as noted previously. Cell viability is a linear relationship with absorbance and was measured at $540 \mathrm{~nm}$. Data demonstrates a decrease in absorbance/cell viability with 2 hours of oxygen and glucose deprivation as compared to control. Though some experimental conditions demonstrate improved viability as compared to oxygen and glucose deprivation, it was not statistically significant. Ten wells were used for each experimental condition. * statisitical difference $(\mathrm{p}<0.005)$ from oxygen and glucose deprivation (OGD); $\dagger$ statisitically equal ( $p>0.999)$ to control. 


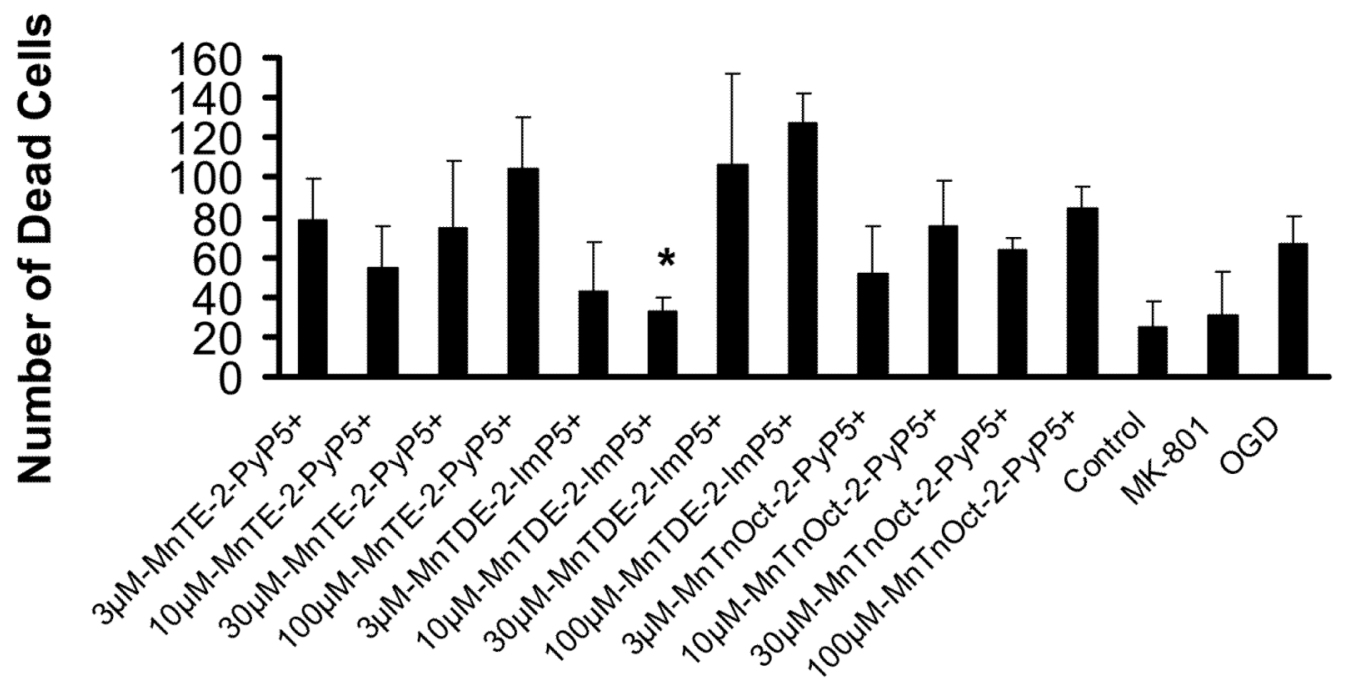

Figure 5. Cell death/propidium iodide assay: $30 \mathrm{~min}$ pretreatment prior to exposure to oxygen and glucose deprivation with continued exposure during 2 hours of oxygen and glucose deprivation

The number of dead cells was counted in three fields in each well and expressed as mean+/standard deviation. Ten wells were examined for each experimental condition. * statisitical difference $(\mathrm{p}<0.005)$ from oxygen and glucose deprivation (OGD); $\dagger$ statisitically equal $(\mathrm{p}>0.999)$ to control. 
Table 1

The properties of Mn porphyhrins: the ability to catalyze dismutation of $\mathrm{O}_{2}{ }^{\cdot-}\left(\mathrm{k}_{\mathrm{cat}}\right)$, the redox ability, i.e. the metal centered redox potential $\left(\mathrm{E}_{1 / 2}\right)$, and the lipophilicity as given by the ratio of the compound and solvent path on TLC silica plates (solvent $\mathrm{KNO}_{3}$ sat $\mathrm{H}_{2} \mathrm{O}: \mathrm{H}_{2} \mathrm{O}$ : acetonitrile= $1: 1+8$ ).

\begin{tabular}{lccc}
\hline Compound & $\operatorname{logk}_{\text {cat }}$ & $\mathbf{E}_{\mathbf{1 / 2}}, \mathbf{m V}$ vs NHE & $\mathbf{R}_{\mathbf{f}}$ \\
\hline MnTE-2-PyP $^{5+} a$ & 7.76 & +228 & 0.13 \\
MnTDE-2-ImP $^{5+} b$ & 7.83 & +346 & 0.17 \\
MnTnHex-2-PyP $^{5+} a$ & 7.48 & +314 & 0.57 \\
MnTnOct-2-PyP $^{5+} a$ & 7.71 & +367 & 0.80 \\
\hline$a_{\text {ref Batinic-Haberle et al, Dalton 2002 }}$ & \\
$b_{\text {ref Batinic-Haberle et al Dalton 2004 }}$
\end{tabular}

